\title{
Microreactor for Clean and Controlled In-situ SEM Imaging of CVD Processes
}

Libor Novak $^{1}$, Petr Wandrol ${ }^{1}$ and Ernst Jan R. Vesseur ${ }^{2}$

${ }^{1}$ Thermo Fisher Scientific, Brno, Jihomoravsky kraj, Czech Republic, ${ }^{2}$ Thermo Fisher Scientific, Eindhoven, Noord-Brabant, Netherlands

We present a method for in-situ SEM imaging of chemical vapor deposition (CVD) processes using a small reactor ( $\mu$ Reactor, $7 \mu$ l volume) positioned within a FIB-SEM chamber (Thermo Scientific Helios G4 UX). To demonstrate the well-controlled conditions within the reactor, we run a graphene monolayer growth process, which is known to require a very clean metal substrate [1]. Polycrystalline platinum foil ( 25 microns thick) was used as the sample from which a small chunk of 50 by 50 micrometers in size was prepared by FIB, transferred on a micromanipulator needle (EasyLift) and glued to the heating plate inside the $\mu$ Reactor [2] by tungsten deposition. The reaction volume was closed by a retractable lid, which assures gas delivery and sealing. In the first step, the platinum sample was annealed at $1000^{\circ} \mathrm{C}$ in a flow of pure hydrogen at $30 \mathrm{~Pa} \mathrm{H}_{2}$ pressure. Hydrogen etching resulted in a pure platinum surface with minimized amount of carbon residuals. In the next step, ethylene was injected to the reactor and mixed with hydrogen. Formation of graphene islands with the typical geometry [3] [4] was observed in-situ in the signal of secondary electrons in immersion mode of Helios FIB-SEM (Fig. 1). The growth kinetics of the graphene layers was controlled by adjusting the partial pressure of ethylene ( $5 \mathrm{~Pa}$ to $10 \mathrm{~Pa}$ ) and by the sample temperature $\left(900^{\circ} \mathrm{C}\right.$ to $\left.1000^{\circ} \mathrm{C}\right)$ [5]. The temperature of the heating plate was calculated from heating wire resistance [6]. The pressure in the reaction volume was estimated from the heating power employing the Pirani gauge principle. In-situ SEM imaging was done using the signal of secondary electrons escaping through an aperture in the reactor lid. The aperture allows for an overpressure of up to several hundreds of pascals inside the reactor, while SEM chamber stays at high vacuum $(<1 \mathrm{E}-2 \mathrm{~Pa})$ supporting standard SEM detection including in-lens. Low inlet flow rates (units of sccm) of the processing gas enable safe operation with reactive gases (pure hydrogen in this case), which can be mixed with inert gas in the pumping system to decrease its concentration below the safe limit at the SEM pump outlet. The small volume of the $\mu$ Reactor and the isolation from the SEM chamber by a pressure limiting aperture assure a process cleanliness that is much higher than in the typical SEM chamber. The design of the retractable lid supports preparation of a clean sample from the bulk using a FIB lift-out in the vacuum of the FIB-SEM system without the need for sample transfer in air before the in-situ experiment.

The successful CVD growth of graphene, which is highly sensitive to the metal substrate cleanliness, shows that the $\mu$ Reactor used on a FIB-SEM tool enables precise control of temperature and gas composition during high resolution SEM imaging of CVD processes. 

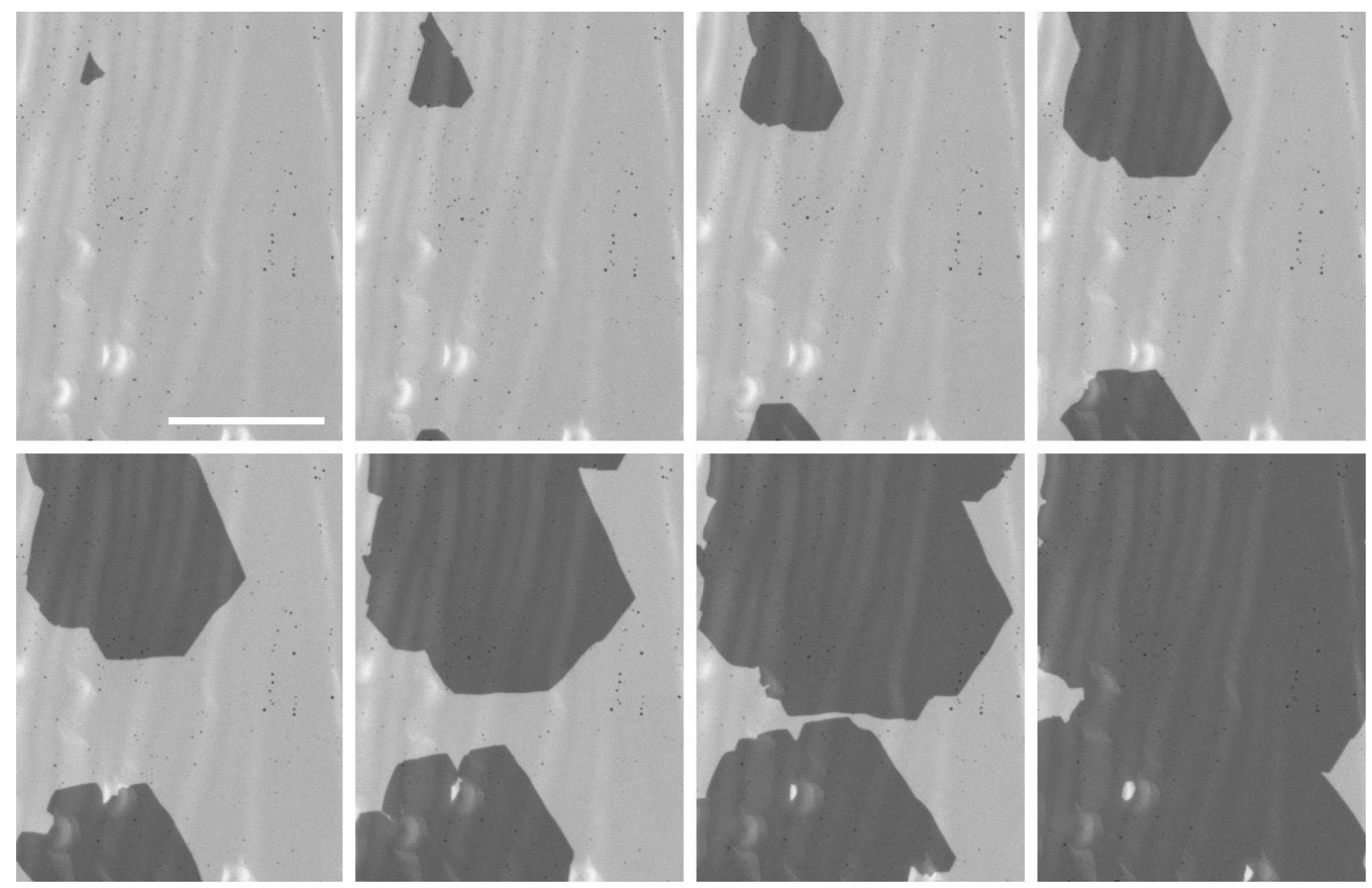

Figure 1. Graphene growth on platinum substrate in $\mu$ Reactor imaged by TLD-SE detector of Helios (UHR mode). Formation of graphene monolayer was stimulated by sample temperature of $900 \mathrm{deg} \mathrm{C}$ and by hydrogen $(25 \mathrm{~Pa})$ and ethylene $(5 \mathrm{~Pa})$ environment. Scale bar length is $5 \mu \mathrm{m}$.

\section{References}

[1] Wang, Z., Dong, J., Cui, Y. et al. Stacking sequence and interlayer coupling in few-layer graphene revealed by in situ imaging. Nat Commun 7, 13256 (2016). https://doi.org/10.1038/ncomms13256

[2] Novak, L., Kolibal, M., Wu, M., Wandrol, P., \& Vystavel, T. Control and in-situ imaging of heat \& gas mediated processes in FIB/SEM system. Microscopy and Microanalysis, 24(S1), 808-809 (2018). doi:10.1017/S1431927618004531

[3] Wang, Z., Dong, J., Li, L. et al. The coalescence behavior of two-dimensional materials revealed by multiscale in situ imaging during chemical vapour deposition growth. ACS Nano 2020 just accepted. https://doi.org/10.1021/acsnano.9b08221

[4] Wang, Z., Weinberg, G., Zhang, Q. et al. Direct observation of graphene growth and associated copper substrate dynamics by in situ scanning electron microscopy. ACS Nano 2015, 9 (2), 1506-1519. https://doi.org/10.1021/nn5059826

[5] https://www.youtube.com/watch?v=GmIfGMcTsn0\&list=PLhJ74CQiOr7bGhmkU2zOKMP-

\section{XXOhnLhOB}

[6] Mele, L. et al. A MEMS-based heating holder for the direct imaging of simultaneous in-situ heating and biasing experiments in scanning/transmission electron microscopes. Microscopy Research and Technique 79 (2016) p. 239. https://doi.org/10.1002/jemt.22623

The authors thank Zhu-Jun Wang for his contribution to the graphene growth experiment. 